\title{
Factors affecting visual outcomes after treatment of infectious endophthalmitis in northeastern Thailand
}

This article was published in the following Dove Press journal:

Clinical Ophthalmology

\section{Yosanan Yospaiboon \\ Anocha Intarapanich \\ Wipada Laovirojjanakul \\ Tanapat Ratanapakorn \\ Suthasinee Sinawat \\ Thuss Sanguansak \\ Chavakij Bhoomibunchoo \\ KKU Eye Center, Department of Ophthalmology, Faculty of Medicine, Khon Kaen University, Khon Kaen, Thailand}

Correspondence: Yosanan Yospaiboon KKU Eye Center, Department of Ophthalmology, Faculty of Medicine, Khon Kaen University, I23 Mitraparb Highway, Khon Kaen 40002, Thailand

Tel +6643348383

Email yosanan@kku.ac.th
Objective: To determine factors affecting visual outcomes after treatment of infectious endophthalmitis during 2012-2016 at a large referral eye center in northeastern Thailand.

Patients and methods: Medical charts of patients with a diagnosis of infectious endophthalmitis including demographic data, types of endophthalmitis, causative organisms, methods of treatment, anatomical, and functional outcomes were retrospectively reviewed. Factors associated with improved visual outcomes were analyzed.

Results: Four hundred and eleven patients (417 eyes) were recruited for the study. The three most common types were post-traumatic $(44.53 \%)$, post-operative $(31.87 \%)$, and endogenous endophthalmitis (17.52\%). Vitreous cultures revealed causative organisms in $41.25 \%$. Most common Gram-positive organisms were coagulase-negative Staphylococcus 30\% (36/120), Bacillus spp. 20\% (24/120), and Streptococcus pneumoniae 18.33\% (20/120). Most common Gram-negatives were Pseudomonas spp. 24.32\% (9/37), Klebsiella spp. 24.32\% (9/37), and Enterobacter spp. $16.21 \%$ (6/37). Methods of treatment were medical treatment $(18.71 \%)$ and surgical treatment (81.29\%), including pars plana vitrectomy with or without silicone oil tamponade $(62.59 \%)$ and destructive surgery (18.71\%). After treatment, visual improvement was noted in $44.6 \%$, stable vision in $18.47 \%$, and worse vision in $36.93 \%$. Factors associated with improved visual outcomes were post-operative endophthalmitis $(P<0.001)$, coagulasenegative Staphylococcus ( $P=0.003)$, and initial visual acuity before treatment of hand motion or better $(P=0.017)$.

Conclusion: Most infectious endophthalmitis patients were post-traumatic, post-operative, and endogenous. The most common method of treatment was pars plana vitrectomy. Treatment could improve visual outcomes and at least stabilize the vision in $63.07 \%$. Factors associated with improved visual outcomes were types of endophthalmitis, causative organisms, and initial visual acuity before treatment.

Keywords: visual outcome, infectious endophthalmitis, prognostic factors

\section{Introduction}

Infectious endophthalmitis is a sight-threatening disease. The etiology may be of exogenous or endogenous causes. It may occur after ocular trauma, intraocular surgery, or spread from adjacent structures or a distant site of infection. ${ }^{1-6}$ Management of infectious endophthalmitis depends on accurate diagnosis and prompt antimicrobial treatment. Causative microorganisms may be bacteria, fungi, or mixed organisms. ${ }^{4-7}$ Microbiological laboratory investigation of the intraocular fluid specimens is, therefore, crucial to the identification of these causative microorganisms. ${ }^{8-10}$ Treatment of infectious endophthalmitis includes specific antimicrobial drugs by various routes such 
as topical, subconjunctival, and intravitreal injection. ${ }^{11,12}$ In the case of medication failure, pars plana vitrectomy with intravitreal antimicrobial injection is a surgical procedure of choice. ${ }^{13-15}$ Usually, endophthalmitis has poor visual outcome and a high rate of visual loss. Advances in vitreoretinal surgery and other additional surgical tools such as silicone oil can, however, improve the final anatomical and functional outcomes. ${ }^{16-18}$ To the best of current knowledge, there has been no study on factors affecting visual prognosis after treatment of the whole spectrum of infectious endophthalmitis. There were only two studies on prognostic factors, one in endogenous endophthalmitis and another in acute postoperative endophthalmitis, and they involved a small sample size. ${ }^{19,20}$ The aim of this study was, therefore, to evaluate the visual outcome of patients with infectious endophthalmitis in a large consecutive series of patients and to determine the factors affecting visual outcomes in these patients.

\section{Patients and methods}

This study follows the tenets of the Declaration of Helsinki, and was approved by the Khon Kaen University Ethics Committee for Human Research (HE 581036). Retrospective medical chart reviews of all patients diagnosed as clinically infectious endophthalmitis at the KKU Eye Center, Department of Ophthalmology, Faculty of Medicine, Khon Kaen University, Khon Kaen, Thailand from 2012 to 2016 were performed. Patient consent to review their medical records was not required by the ethics committee, since the case report forms had no linkage to the patient identities and the researchers respected the privacy of the patients. Inclusion criteria were those who had a clinical diagnosis of infectious endophthalmitis, history of prior intraocular injury or surgery no longer than 6 weeks before, had medical or surgical treatment at the hospital, and a follow-up period of at least 6 weeks. The patients who had a history of corneal disorders or retinal diseases, which might interfere with visual acuity, were excluded from the study. Those who had active glaucoma and uncontrolled diabetes mellitus were also excluded. The collected data including age, gender, occupation, underlying diseases, history of ocular injury and intraocular surgery, initial and final best-corrected visual acuity (BCVA) examinations, the treatment procedures, and anatomical and functional outcomes. Causative organisms identified were also recorded.

Microbiological investigations of vitreous fluids were done in all patients. Undiluted vitreous specimens were obtained by vitreous aspiration through a 23-gauge needle. The vitreous specimens were inoculated on blood agar, chocolate agar, MacConkey agar, and Sabouraud dextrose agar, as well as in thioglycolate broth and blood culture bottles. The causative organisms were then identified and reported. In the case of endogenous endophthalmitis, hemocultures or cultures from extraocular infection sites were also performed.

The medical treatments consisted of intravitreal vancomycin $(1 \mathrm{mg} / 0.1 \mathrm{~mL})$ and ceftazidime $(2.25 \mathrm{mg} / 0.1 \mathrm{~mL})$. Intensive topical and systemic antibiotics were also included. Surgical treatment consisted of pars plana vitrectomy and intravitreal antibiotics. This was indicated in patients with the loss of red reflex and substantial vitreous opacity on ultrasonography. In severe cases, pars plana vitrectomy combined with silicone oil tamponade was performed, depending on the decisions of the surgeons.

The primary outcomes were factors affecting the visual outcomes after treatment. The patients were classified as "improved" visual outcome when their final BCVA was one or more Snellen acuity line better than initial BCVA. They were classified as "worse" when final BCVA was one or more Snellen acuity line worse than initial BCVA. Stable visual outcome was defined when final BCVA did not change from initial BCVA. The secondary outcomes were types of infectious endophthalmitis, types of causative organisms, and types of treatment modalities.

Statistical analysis was performed using STATA version 10.0 (StataCorp LP, College Station, TX, USA). The categorical data, such as gender, affected eyes, underlying diseases, types of endophthalmitis, treatment procedures, and visual outcomes, are shown in numbers and percentages. The numerical data, such as numbers of positive cultures and causative organisms, are also reported in numbers and percentages. Age is shown as mean \pm standard deviation and range. Visual outcomes after treatment in different patient groups were compared and analyzed using chi-square test and odds ratios with $95 \%$ confidence intervals. The differences were considered significant when the $P$-value was $<0.05$.

\section{Results}

A total of 411 patients and 417 eyes with clinically infectious endophthalmitis were recruited into the study. There were 287 male and 124 female patients. The mean age was $50.99 \pm 20.76$ years and ranged from $18-89$ years. Two hundred and eighteen (53.04\%) right eyes and 187 (45.50\%) left eyes were affected. There were six cases $(1.46 \%)$ having both eyes affected. The types of endophthalmitis in this study are shown in Table 1. One hundred and eighty-three patients had a history of ocular trauma before the infection. One hundred and thirty-two patients developed infection after intraocular surgery. Seventy-two patients were diagnosed with endogenous endophthalmitis by evidence of extraocular infection sites. 
Table I Types of endophthalmitis in 4 I I patients in the study

\begin{tabular}{lll}
\hline Types of endophthalmitis & No & Percentage \\
\hline Post-traumatic & 183 & 44.53 \\
Post-operative & 132 & 32.12 \\
Endogenous & 72 & 17.52 \\
Keratitis-associated & 16 & 3.89 \\
Bleb-related & 5 & 1.22 \\
Post-intravitreal injection & 3 & 0.72 \\
Total & 411 & 100.00 \\
\hline
\end{tabular}

Positive cultures were found in 172 (41.25\%) of 417 eyes. Bacteria were isolated in 157 eyes $(91.28 \%)$, fungi in eight eyes $(4.65 \%)$, and mixed organisms in seven eyes (4.07\%). The data on specific causative microorganisms are summarized in Table 2. There were Gram positives in
120 eyes (76.43\%) and Gram negatives in 37 eyes (23.57\%). The most frequent bacteria were coagulase-negative Staphylococcus (36), Bacillus spp. (24), Streptococcus pneumonia (20), $\beta$-hemolytic streptococcus (12), Pseudomonas spp. (9), Klebsiella spp. (9), and Enterococcus spp. (8), in descending order of frequency. It is interesting that the most common organism in post-traumatic endophthalmitis was Bacillus spp., whereas coagulase-negative Staphylococcus was the most frequent organism in post-operative type. Group B $\beta$ hemolytic Streptococcus, Klebsiella spp., and Escherichia coli were the three most common organisms identified in patients with endogenous endophthalmitis. It is also noted from Table 2 that Pseudomonas aeruginosa was commonly identified in endophthalmitis associated with keratitis, and

Table 2 Number of causative organisms found in positive cultures of 172 eyes

\begin{tabular}{|c|c|c|c|c|c|c|c|}
\hline Organisms & $\begin{array}{l}\text { Post- } \\
\text { trauma }\end{array}$ & $\begin{array}{l}\text { Post- } \\
\text { operative }\end{array}$ & Endogenous & $\begin{array}{l}\text { Keratitis } \\
\text { associated }\end{array}$ & $\begin{array}{l}\text { Bleb- } \\
\text { related }\end{array}$ & $\begin{array}{l}\text { Post- } \\
\text { injection }\end{array}$ & Total \\
\hline Bacteria & & & & & & & 157 \\
\hline Gram-positive & & & & & & & 120 \\
\hline Coagulase-negative Staphylococcus & 9 & 21 & 4 & - & - & 2 & 36 \\
\hline Bacillus spp. & 23 & 1 & - & - & - & - & 24 \\
\hline Streptococcus pneumonia & 4 & 11 & 3 & 2 & - & - & 20 \\
\hline$\beta$-Hemolytic Streptococcus & - & 1 & 11 & - & - & - & 12 \\
\hline Enterococcus spp. & 4 & 2 & I & & I & & 8 \\
\hline Streptococcus viridans & 3 & 4 & - & - & - & - & 7 \\
\hline Corynebacterium spp. & 4 & 1 & - & 1 & - & - & 6 \\
\hline Streptococcus suis & - & - & 2 & - & - & - & 2 \\
\hline Streptococcus spp. & - & - & - & - & 2 & - & 1 \\
\hline$\beta$-Nonhemolytic Strepcoccus & - & - & 1 & - & - & - & I \\
\hline Nocardia spp. & 1 & - & - & - & - & - & I \\
\hline Micrococcus spp. & - & - & - & 1 & - & - & I \\
\hline Propionibacterium acne & - & 1 & - & - & - & - & I \\
\hline Gram negative & & & & & & & 37 \\
\hline Pseudomonas aeruginosa & 5 & - & - & 4 & - & - & 9 \\
\hline Klebsiella spp. & - & 1 & 8 & - & - & - & 9 \\
\hline Enterobacter spp. & 3 & 1 & 1 & - & 1 & - & 6 \\
\hline Escherichia coli & - & 1 & 4 & - & - & - & 5 \\
\hline Stenotrophomonas spp. & - & - & 1 & - & - & - & I \\
\hline Haemophilus parainfluenzae & - & 1 & - & - & - & - & I \\
\hline Serratia spp. & - & - & - & - & I & - & 1 \\
\hline Burkholderia spp. & - & 1 & - & - & - & - & I \\
\hline Bordetella spp. & 1 & - & - & - & - & - & 1 \\
\hline Acinetobacter baumannii & - & 1 & - & - & - & - & I \\
\hline Aeromonas hydrophila & 1 & - & - & - & - & - & 1 \\
\hline Fungus & & & & & & & 8 \\
\hline Aspergillus spp. & 1 & 3 & 1 & 1 & - & - & 6 \\
\hline Fusarium spp. & I & - & - & I & - & - & 2 \\
\hline Mixed organisms & & & & & & & 7 \\
\hline Bacillus spp. + coagulase-negative Staphylococcus & I & 1 & - & - & - & - & 2 \\
\hline Bacillus spp. + Enterobacter spp. + E. coli & I & - & - & - & - & - & 1 \\
\hline Bacillus spp. + Curvularia spp. & 1 & - & - & - & - & - & I \\
\hline S. viridans + Enterobacter spp. + E. coli & 1 & - & - & - & - & - & I \\
\hline S. viridans + Aspergillus spp. & 1 & - & - & - & - & - & I \\
\hline Aspergillus spp. + Penicillium spp. & 1 & - & - & - & - & - & 1 \\
\hline
\end{tabular}


Table 3 Treatment modalities of 417 eyes in the study

\begin{tabular}{lll}
\hline Treatment modalities & No & Percentage \\
\hline Medical treatment & 78 & 18.71 \\
Pars plana vitrectomy & 174 & 41.72 \\
Par plana vitrectomy with silicone oil & 87 & 20.86 \\
Destructive surgery & 78 & 18.71 \\
Total & 417 & 100.00 \\
\hline
\end{tabular}

Streptococcus spp. was common in bleb-related infection. In addition, almost all patients with mixed organisms developed endophthalmitis after ocular trauma.

Treatment modalities, including medical treatment, pars plans vitrectomy with or without silicone oil tamponade, and destructive surgery (evisceration or enucleation) are shown in Table 3. The most common method of treatment was pars plana vitrectomy (261 eyes, 62.29\%). Infection was controlled by medical treatment in 78 eyes $(18.71 \%)$, and there were 78 eyes (18.71\%) ending up with evisceration or enucleation.

The initial visual acuities and final visual outcomes are compared in Figure 1. The majority of patients (332/417, $79.62 \%$ ) had initial visual acuity of hand motion (191), light perception (93), or no light perception (48). Final visual outcomes that are reported as improved, stable, and worse are shown in Figures 2-4. In addition to 78 eyes (18.71\%) with eye removal, the visual acuities were improved in 186 eyes (44.6\%), stable in 77 eyes (18.47\%), and worse in 76 eyes $(18.22 \%)$. It is noted that treatment could improve visual outcome and at least stabilize the vision in 263 eyes (63.07\%).
The improved visual outcomes according to the type of endophthalmitis, treatment modalities, and initial visual acuity before treatment are also shown in Figures 2-4.

Factors affecting the visual outcomes are demonstrated in Table 4. It revealed that the types of endophthalmitis, types of causative organisms, and initial visual acuity are factors associated with improved visual outcome with statistically significant differences. Post-operative endophthalmitis had the most favorable visual outcome when compared to endogenous and bleb-related types $(P<0.001)$. Coagulase-negative Staphylococcus had the most favorable visual outcome when compared to Bacillus, Streptococcus, and mixed infections $(P=0.003)$. The initial vision capable of counting fingers had the most favorable visual outcome when compared to initial visual acuity of light perception $(P=0.017)$.

\section{Discussion}

The most common type of infectious endophthalmitis in this study was post-traumatic endophthalmitis. This was not consistent with the reviewed literatures that reported postoperative cause as the most common type. ${ }^{4,6,21}$ This study, however, agrees with previous studies in Thailand. ${ }^{5,8,9}$ This may be attributed to a higher incidence of ocular trauma, especially with vegetative material, in the rural areas. Postoperative endophthalmitis was the next most common and similar to other previous studies, where most of the patients developed infection after cataract surgery. ${ }^{4,5,21}$

In all types of endophthalmitis, accurate diagnosis should be accomplished by clinical and microbiological laboratory

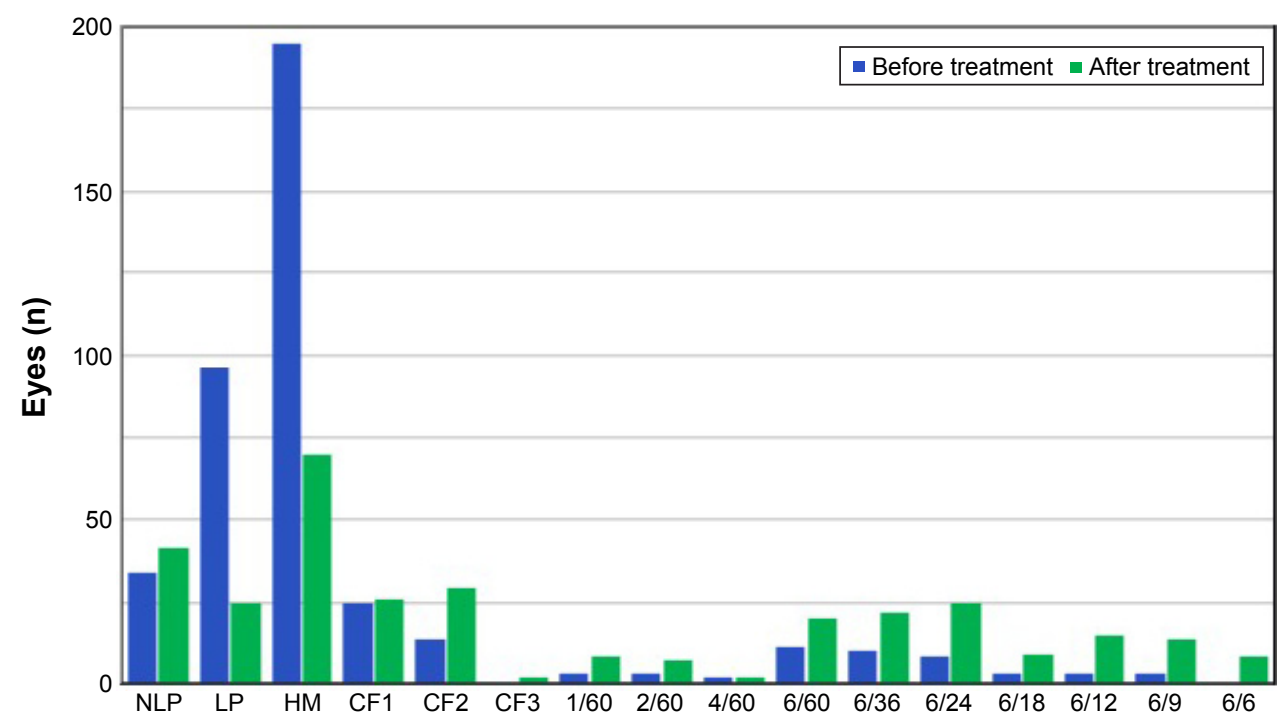

Figure I Initial best-corrected visual acuity before and after treatment.

Abbreviations: NLP, no light perception; LP, light perception; HM, hand motion; CFI, counting finger at I foot; CF2, counting finger at 2 feet; CF3, counting finger at 3 feet. 


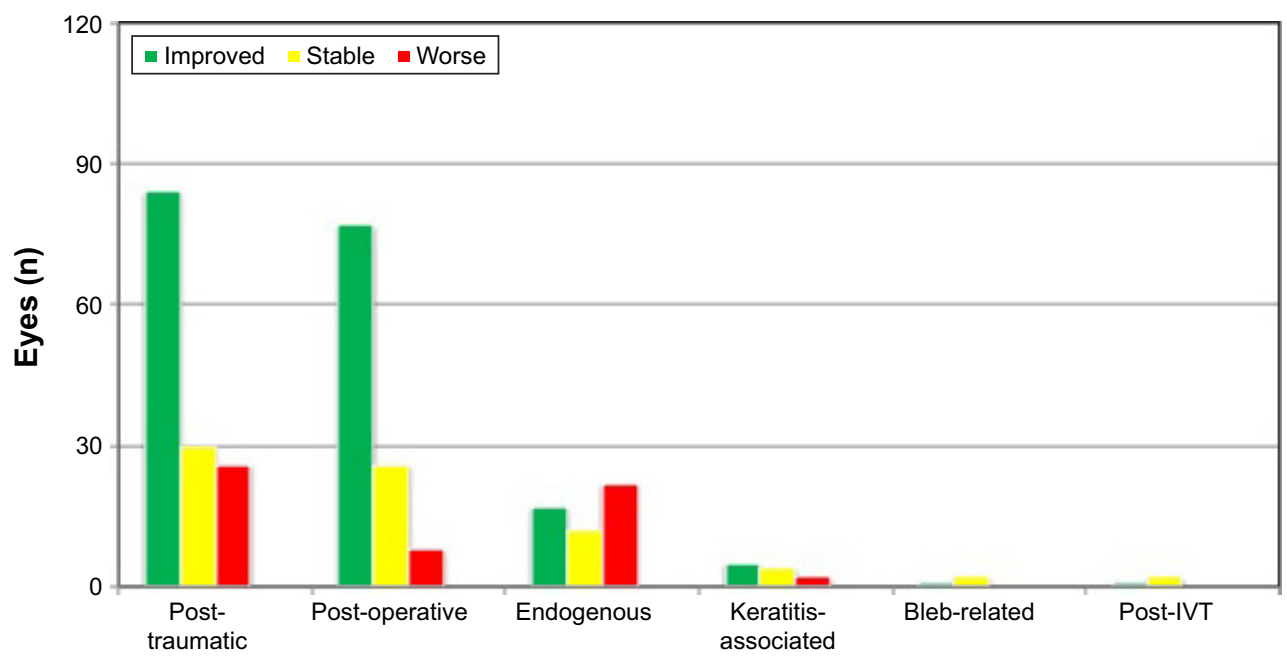

Figure 2 Visual outcomes vs different types of endophthalmitis.

Abbreviation: IVT, intravitreal injection.

investigation. Smears and cultures of intraocular fluid specimens for identification of the causative organisms are still used as a gold standard for diagnosis. In the present study, both conventional culture media and blood culture bottles were used for culture of the vitreous fluid specimens, because this laboratory's previous studies revealed that vitreous culture in blood culture bottles yielded more positive results than conventional culture media, and a combination of both improved the possibility of a positive culture. ${ }^{8,9}$ The culture yield in this study was only $41.25 \%$. This may be attributed to many factors. The possibility may be that the patients had been partially treated with antimicrobial therapy before referral to the KKU Eye Center. Another reason may be that some causative organisms need specific media or conditions for growth that were not provided in the study.

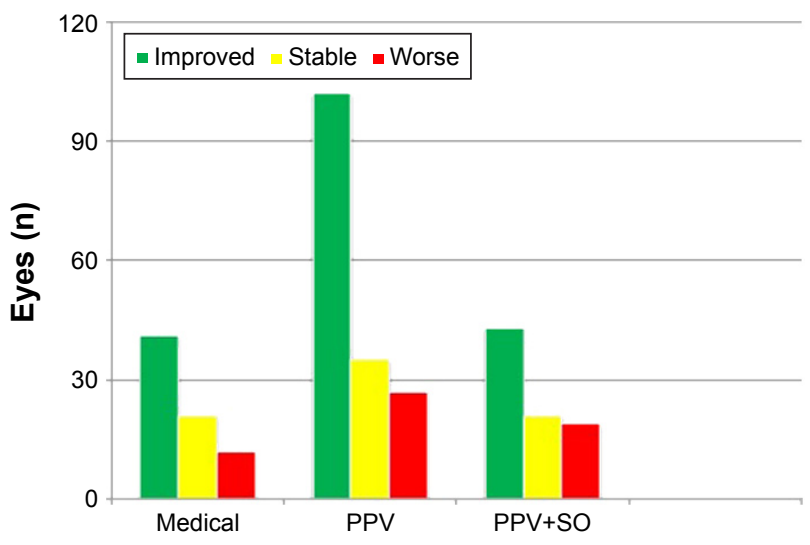

Figure 3 Visual outcomes vs different treatment modalities. Abbreviations: PPV, pars plana vitrectomy; PPV+SO, pars plana vitrectomy and silicone oil tamponade.
In this study, the most common type of organisms were Gram-positive bacteria, and the majority of them was coagulase-negative Staphylococcus. This result agrees well with other previous studies. ${ }^{4,5,8,20,21}$ The next most common Gram-positive bacteria were Bacillus spp. and Streptococcus pneumoniae. This finding is also similar to other previous studies. ${ }^{5,8,9}$ The three most common Gram-negative bacteria were Pseudomonas aeruginosa, Klebsiella spp., and Enterobacter spp. This is also in agreement with previous reports. ${ }^{5,9}$ There were eight eyes with fungal endophthalmitis. Six of them were Aspergillus spp. This is in contrast to a previous study that reported Candida spp. as the most frequent fungus isolates. ${ }^{21}$ In addition, there were seven eyes with endophthalmitis caused by mixed organisms. Most of them developed endophthalmitis after ocular trauma.

Factors associated with an improved visual outcome were analyzed. When compared to other types of endophthalmitis, the post-operative type had the most favorable visual outcome (Figure 2 and Table 4). This may be attributed to the awareness of both patients and ophthalmologists, so that most patients had early diagnosis and treatment. Fortunately, the causative organisms were mostly coagulase-negative Staphylococcus, which is less virulent (Table 2). Posttraumatic endophthalmitis usually has virulent Bacillus and mixed infections that result in a poor visual prognosis. ${ }^{22-24}$ The visual outcome of endogenous endophthalmitis is rather poor, due to the virulence of the organisms and compromised host conditions. ${ }^{19}$

Regarding causative organisms, patients with coagulase-negative Staphylococcus endophthalmitis had more improved visual outcomes than other organisms with a 


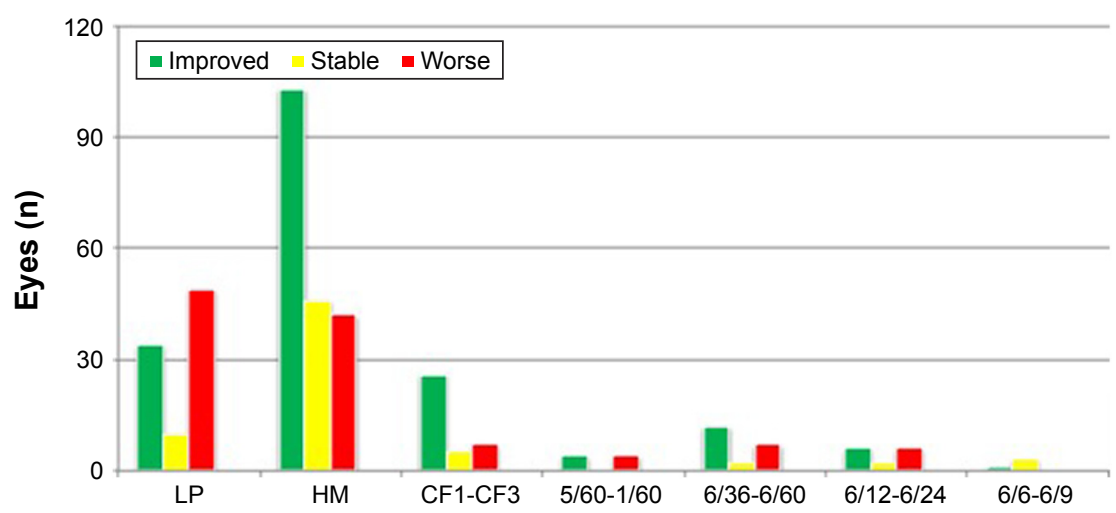

Figure 4 Visual outcomes vs initial visual acuity before treatment.

Abbreviations: LP, light perception; HM, hand motion; CFI, counting finger at I foot; CF3, counting finger at 3 feet.

statistically significant difference. This finding is similar to a previous report. ${ }^{20}$ When compared to other Gram-positive bacterial infections, patients with Bacillus and Streptococcus endophthalmitis seemed to have a poor visual prognosis.
Mixed infections with Bacillus, Gram-negative bacteria, or fungi in post-traumatic patients were associated with the most unfavorable visual outcomes. This is also consistent with other previous studies. ${ }^{22-24}$ In this study, mixed organisms,

Table 4 Factors associated with improved visual outcome

\begin{tabular}{|c|c|c|c|}
\hline Factors & $\begin{array}{l}\text { Improved visual } \\
\text { outcome, } \mathbf{n}(\%)\end{array}$ & $\begin{array}{l}\text { Odds ratio } \\
(95 \% \mathrm{Cl})\end{array}$ & $P$-value \\
\hline Types of endophthalmitis & & & $<0.001$ \\
\hline Post-operative & $78 / 132(59.09)$ & $5.60(0.6 I-5 I .48)$ & \\
\hline Post-traumatic & $84 / 183(45.90)$ & $3.39(0.37-30.95)$ & \\
\hline Post-intravitreal injection & $\mathrm{I} / 3(33.33)$ & $2.00(0.08-51.59)$ & \\
\hline Post-keratitis & $5 / 16(31.25)$ & $1.82(0.16-20.7 I)$ & \\
\hline Endogenous & $17 / 72(23.61)$ & $1.24(0.13-11.82)$ & \\
\hline Bleb-related & $\mathrm{I} / 5(20)$ & 1.00 & \\
\hline Types of organisms & & & 0.003 \\
\hline \multicolumn{4}{|l|}{ Gram positive } \\
\hline Coagulase-negative Staphylococcus & $24 / 36(66.67)$ & $12.00(|.29-||| .32)$ & \\
\hline Bacillus spp. & $5 / 24(20.83)$ & $1.58(0.15-16.31)$ & \\
\hline Streptococcus pneumoniae & $3 / 20(15.00)$ & $1.06(0.09-12.23)$ & \\
\hline Other Gram positive & $16 / 40(40.00)$ & $4.00(0.44-36.44)$ & \\
\hline \multicolumn{4}{|l|}{ Gram negative } \\
\hline Pseudomonas spp. & $3 / 9(33.33)$ & $3.00(0.24-37.67)$ & \\
\hline Klebsiella spp. & $2 / 9(22.22)$ & $1.71(0.12-23.94)$ & \\
\hline Escherichia coli & I/5 (20.00) & $1.50(0.07-31.57)$ & \\
\hline Other Gram negative & $4 / 14(28.57)$ & $2.40(0.21-26.82)$ & \\
\hline Fungus & $2 / 8(25)$ & $2.00(0.14-28.42)$ & \\
\hline Mixed organisms & I/7 (14.29) & 1.00 & \\
\hline Treatment modalities & & & 0.26 \\
\hline Medical treatment & $4 \mathrm{I} / 78(52.56)$ & $1.16(0.62-2.17)$ & \\
\hline PPV & $102 / 174(58.62)$ & $1.53(0.90-2.61)$ & \\
\hline PPV with silicone oil & $43 / 87(49.43)$ & 1.00 & \\
\hline Initial visual acuity & & & 0.017 \\
\hline $6 / 6-6 / 9$ & I/4 (25) & $0.58(0.06-5.78)$ & \\
\hline $6 / 12-6 / 24$ & $6 / 14(42.86)$ & $1.30(0.42-4.07)$ & \\
\hline $6 / 36-6 / 60$ & $|2 / 2|(57.14)$ & $2.31(0.88-6.05)$ & \\
\hline $5 / 60-1 / 60$ & $4 / 8(50)$ & I.73 $(0.4 \mid-7.39)$ & \\
\hline Counting fingers & $26 / 38(68.42)$ & $3.74(1.68-8.40)$ & \\
\hline Hand motion & $103 / 19 \mid(53.93)$ & $2.03(1.22-3.38)$ & \\
\hline Light perception & $34 / 93(36.56)$ & 1.00 & \\
\hline
\end{tabular}

Abbreviations: $\mathrm{n}$, number; $\mathrm{Cl}$, confidence interval; PPV, pars plana vitrectomy. 
either mixed bacterial or mixed bacterial and fungal infections, had the poorest visual prognosis. Only one of seven eyes had vision improvement, three eyes had a worse visual outcome, and three eyes had evisceration or enucleation.

In this study, pars plana vitrectomy had a slightly more improved visual outcome than other treatment modalities, but the difference was not statistically significant (Figure 3 and Table 4). Although vitrectomy seems to be the best treatment modality, because it removes the bulk of organism loads and facilitates the distribution of intravitreal antibiotics, ${ }^{13-15}$ vitrectomy cannot be compared with other methods of treatment in this study, since patients in each treatment group had different degrees of severity. Patients in the medical treatment group usually had a mild degree of infection, whereas those in the surgical treatment group had more severe clinical signs of infection. This may be one of the reasons why there is no significant difference in the association.

Initial visual acuity before treatment was also associated with improved visual outcome. Initial vision of hand motion or better had a more favorable visual outcome than the initial visual acuity of only light perception, with a statistically significant difference (Figure 4 and Table 4). Patients with only light perception before treatment had the worst visual prognosis. They only had a one-third chance of improved visual outcome (34 eyes), whereas 11 eyes had stable vision, and most of them (48 eyes) had worse vision (34 of 48 eyes were eviscerated or enucleated). It is also noted that there were four patients with 6/9 vision before treatment. Although between them they had only one eye (25\%) with improved visual outcome, all of the remaining three eyes had stable vision. This indicates that all of them preserved their initial vision.

\section{Conclusion}

Visual outcomes after treatment of patients with infectious endophthalmitis have been studied. Most patients in this study had post-traumatic endophthalmitis. The most common causative organisms were Gram-positive bacteria, particularly coagulase-negative Staphylococcus. Factors associated with improved visual outcome have been analyzed. Post-operative type of endophthalmitis, coagulase-negative Staphylococcus, and initial visual acuity of hand motion or better were regarded as important factors associated with improved visual outcome. Although infectious endophthalmitis is a devastating eye disease, and treatment could not change the virulence of causative organisms and its poor visual outcome, this study demonstrated that microbiological laboratory investigation of vitreous specimens and prompt specific antimicrobial therapy and/or surgical treatment could improve or at least stabilize some useful vision, even in patients with poor initial visual acuity.

The strength of this study was a large series of patients. An obvious limitation was that it was a retrospective study. There might be some important data not recorded in the medical charts. In this study, data on time to presentation and the presence of relative afferent pupillary defects are missing. Another limitation was that the number of patients in each subgroup might be too small for statistically significant differences and conclusion. It is recommended that further prospective studies with larger sample sizes should be pursued.

\section{Acknowledgments}

The authors thank Dr Kaewjai Thepsuthammarat, Clinical Epidemiology Unit, Faculty of Medicine, Khon Kaen University for statistical analysis and Professor James A. Will, University of Wisconsin, USA for editing the manuscript via the Faculty of Medicine Publication Clinic, Khon Kaen University, Thailand. This study was supported by invitation research grant (IN5822) from the Faculty of Medicine, Khon Kaen University and was presented in part at the 10th Asia Pacific Congress of Vitreo-Retina Society, December 8-10, 2016, Bangkok, Thailand.

\section{Disclosure}

The authors report no conflicts of interest in this work.

\section{References}

1. Vaziri K, Schwartz SG, Kishor K, Flynn HW Jr. Endophthalmitis: state of the art. Clin Ophthalmol. 2015;9:95-108.

2. Bhagat N, Nagori S, Zarbin M. Post-traumatic infectious endophthalmitis Surv Ophthalmol. 2011;56(3):214-251.

3. Pathengay A, Flynn HW Jr, Isom RF, Miller D. Endophthalmitis outbreaks following cataract surgery: causative organisms, etiologies, and visual acuity outcomes. J Cataract Refract Surg. 2012;38(7):1278-1282.

4. Karacal H, Kymes SM, Apte RS. Retrospective analysis of etiopathogenesis of all cases of endophthalmitis at a large tertiary referral center. Int Ophthalmol. 2007;27:251-259.

5. Bhoomibunchoo C, Ratanapakorn T, Sinawat S, Sanguansak T, Moontawee K, Yospaiboon Y. Infectious endophthalmitis: review of 420 cases. Clin Ophthalmol. 2013;7:247-252.

6. Kresloff MS, Castellarin AA, Zarbin MA. Endophthalmitis. Surv Ophthalmol. 1998;43:195-224.

7. Silva RA, Sridhar J, Miller D, Wykoff CC, Flynn HW Jr. Exogenous fungal endophthalmitis: an analysis of isolates and susceptibilities to antifungal agents over a 20-year period (1990-2010). Am J Ophthalmol. 2015;159(2):257-264.

8. Yospaiboon Y, Saree S, Pasadhika S. Blood culture and conventional culture media for vitreous culture in endophthalmitis. J Med Assoc Thai. 2005;88(5):639-642

9. Thariya P, Yospaiboon Y, Sinawat S, Sanguansak T, Bhoomibunchoo C, Laovirojjanakul W. Blood culture bottles are superior to conventional media for vitreous culture. Clin Exp Ophthalmol. 2016;44(6):488-491. 
10. Ness T, Pelz K. [Endophthalmitis: improvement of culture results]. Ophthalmologe. 2000;97:33-37.

11. O'Day DM, Jones DB, Patrineli J, Elliott JH. Staphylococcus epidermidis endophthalmitis: visual outcome following non-invasive therapy. Ophthalmology. 1982;89:354-360.

12. López-Cabezas C, Muner DS, Massa MR, Mensa Pueyo JM. Antibiotics in endophthalmitis: microbiological and pharmacokinetic considerations. Curr Clin Pharmacol. 2010;5(1):47-54.

13. Callegan MC, Guess S, Wheatley NR, et al. Efficacy of vitrectomy in improving the outcome of Bacillus cereus endophthalmitis. Retina. 2011;31(8):1518-1524.

14. Recchia FM, Scott IU, Brown GC, Brown MM, Ho AC, Ip MS. Smallgauge pars plana vitrectomy: a report by the American Academy of Ophthalmology. Ophthalmology. 2010;117(9):1851-1857.

15. Ng JQ, Morlet N, Pearman JW, Constable IJ, McAllister IL, Kennedy CJ, et al. Management and outcomes of postoperative endophthalmitis since the endophthalmitis vitrectomy study: the Endophthalmitis Population Study of Western Australia (EPSWA)'s fifth report. Ophthalmology. 2005;112:1199-1206.

16. Do T, Hon DN, Aung T, Hien ND, Cowan CL Jr. Bacterial endogenous endophthalmitis in Vietnam: a randomized controlled trial comparing vitrectomy with silicone oil versus vitrectomy alone. Clin Ophthalmol. 2014;8:1633-1640.

17. Pinarci EY, Yesilirmak N, Bayar SA, Sizmaz S, Akkoyun I, Yilmaz G. The results of pars plana vitrectomy and silicone oil tamponade for endophthalmitis after intravitreal injections. Int Ophthalmol. 2013;33(4): 361-365.
18. Azad R, Ravi K, Talwar D, Rajpal, Kumar N. Pars planar vitrectomy with or without silicone oil endotamponade in post-traumatic endophthalmitis. Graefes Arch Clin Exp Ophthalmol. 2003;241(6):478-483.

19. Sallam A, Taylor SR, Khan A, et al. Factors determining visual outcome in endogenous endophthalmitis. Retina. 2012;32(6):1129-1134.

20. Combey de Lambert A, Campolmi N, Cornut PL, et al. Baseline factors predictive of visual prognosis in acute postoperative bacterial endophthalmitis in patients undergoing cataract surgery. JAMA Ophthalmol. 2013;131(9):1159-1166.

21. Krause L, Bechrakis NE, Heimann H, Kildal D, Foerster MH. Incidence and outcome of endophthalmitis over a 13-year period. Can $J$ Ophthalmol. 2009;44(1):88-94.

22. Jindal A, Moreker MR, Pathengay A, et al. Polymicrobial endophthalmitis: prevalence, causative organisms and visual outcome. J Ophthalmic Inflamm Infect. 2013;3(1):6.

23. Dave VP, Pathengay A, Budhiraja I, et al. Clinical presentation, microbiologic profile and factors predicting outcomes in Bacillus endophthalmitis. Retina. 2017. [Epub ahead of print]

24. Das T, Choudhury K, Sharma S, Jalali S, Nuthethi R; Endophthalmitis Research Group. Clinical profile and outcome in Bacillus endophthalmitis. Ophthalmology. 2001;108(10):1819-1825.
Clinical Ophthalmology

\section{Publish your work in this journal}

Clinical Ophthalmology is an international, peer-reviewed journal covering all subspecialties within ophthalmology. Key topics include: Optometry; Visual science; Pharmacology and drug therapy in eye diseases; Basic Sciences; Primary and Secondary eye care; Patient Safety and Quality of Care Improvements. This journal is indexed on

\section{Dovepress}

PubMed Central and CAS, and is the official journal of The Society of Clinical Ophthalmology (SCO). The manuscript management system is completely online and includes a very quick and fair peer-review system, which is all easy to use. Visit http://www.dovepress.com/ testimonials.php to read real quotes from published authors. 